\title{
Efficacy and safety of Sofosbuvir-containing regimens in patients co-infected with chronic hepatitis $C$ virus and human immunodeficiency virus: a meta-analysis
}

\author{
Guotao Li ${ }^{1}$, Ke Zang ${ }^{1}$, Guoqiang Zhang ${ }^{1}$, Danyan Zhu' ${ }^{1}$ and Xiaozhao Deng ${ }^{2^{*}}$ (I)
}

\begin{abstract}
Background: The treatment of hepatitis $\mathrm{C}$ virus (HCV) in HCV/human immunodeficiency virus (HIV) co-infected patients remains complex. This present meta-analysis evaluated the efficacy and safety of Sofosbuvir (SOF) for treatment in HCV/HIV co-infected patients using the most recent and available data.

Methods: A systematic search of the published data was conducted in PubMed Medline, EMBASE and Cochrane databases. Eligible studies were clinical trials, case-control studies or prospective cohort studies aiming at assessing the efficacy and safety of the SOF-containing regimens in patients co-infected with HCV and HIV. Heterogeneity of results was assessed and a pooled analysis was performed using random effects model with maximum likelihood estimate and 95\% confidence intervals $(95 \% \mathrm{Cl})$. Subgroup analysis and assessment of publication bias through Egger's test were also performed. STATA 13.0 software was used to analyze the data.
\end{abstract}

Results: Seven studies ( $n=1167$ co-infected patients) were included in this analysis. The pooled estimate of sustained virological response at 12 weeks (SVR12) was 94.0\% (95\%Cl: 92.0\%-95.0\%). Subgroup analysis showed that the treatment-naive patients had higher SVR12 compared with patients that were treated before $\left(X^{2}=21.39, P<0.01\right)$. The pooled incidence of any adverse events (AEs) was 79.6\% (95\%Cl: 77.1\%-82.1\%). Publication bias did not exist.

Conclusion: The results of this study showed that the treatment response of SOF-containing regimens in patients co-infected with HIV and HCV was satisfied. Attention should be paid to the high rates of AEs.

Keywords: Sofosbuvir, HCV/HIV co-infection, SVR12, Meta-analysis

\section{Background}

It's estimated that up to 7 million patients are infected with both human immunodeficiency virus (HIV) and hepatitis $\mathrm{C}$ virus (HCV) worldwide [1]. As HIV and HCV share similar route of transmission, serious liver disease caused by HCV has emerged as one important cause of non-AIDS associated morbidity and mortality in co-infected patients [2-4]. Eradication of HCV in co-infected patients has relation to the reduction in development of liver disease, HIV progression and mortality not related to liver disease [5]. The

\footnotetext{
* Correspondence: molescience@163.com

${ }^{2}$ HuaDong Research Institute for Medicine and Biotecnics, No.293 Zhongshan East Road, Nanjing 210002, China

Full list of author information is available at the end of the article
}

development of direct acting antivirals (DAAs) for treatment of $\mathrm{HCV}$ has been eagerly awaited to improve $\mathrm{HCV}$ treatment. Though numerous new DAAs are being developed, treatment of $\mathrm{HCV}$ in $\mathrm{HCV} / \mathrm{HIV}$ co-infected patients remains complex with challenges including drugdrug interactions between HIV drugs and HCV protease inhibitors, high rates of adverse events (AEs), high pill burden and long treatment duration [6]. Sofosbuvir (SOF) which is an oral nucleotide analogue inhibitor of the HCV non-structural 5B (NS5B) polymerase has recently been approved for treatment of patients infected of $\mathrm{HCV}$ genotypes 1-4 [7, 8]. As SOF has minimal drug interactions with DAAs, clinical studies supporting the use of SOF in combination with other DAAs for the treatment of $\mathrm{HCV} / \mathrm{HIV}$ co-infected patients were emerging. A few 
studies of them have performed post-hoc analyses to evaluate the efficacy and safety of SOF with or without other DAAs in patients with certain characteristics. However, a systematic review with a comprehensive comparison of the outcome data identified by the clinical studies is still not available. This study aims at summarizing the currently available data on treatment of HCV in HIV with SOF and to provide guidance in practical clinical algorithms of HCV/HIV co-infected patients' management.

\section{Methods}

\section{Searching strategy and selection of literature}

Three electronic database, including PubMed, EMBASE and Cochrane databases were searched for studies. The literature search was performed using the following terms: "hepatitis C" or "HCV" or "hepacivir"; "sofodbuvir" or "Sovaldi" or "SOF" or "GS-7977" or "PSI-7977"; "HIV" or "AIDS" or "human immunodeficiency virus". We also performed manual search through checking the references of included studies and published narrative reviews for potentially eligible studies.

Clinical trials, case-control studies or prospective cohort studies aiming at assessing the efficacy and safety of the SOF-containing regimens in patients co-infected with HCV and HIV were included. Published or unpublished studies were enrolled if they met the following criteria: (1) the study population were co-infected with HCV and HIV; (2) interventions included SOF; (3) the main outcome measure was SVR12; (4) the studies reported the number of patients who achieved and failed to achieve SVR12; (5) the studies showed the results of safety outcome. Studies were excluded if they met any of the following criteria: (1) participants were co-infected with other virus; (2) the main outcome measure was not SVR12; (3) studies failed to report the main outcome; (4) conference abstracts without full text.

\section{Study selection and data extraction}

Two reviewers ZK and ZDY independently selected articles potentially eligible for inclusion by screening titles, abstracts of each article. Then they tried their best to get the full texts and assessed the eligibility of the previous selected articles by reviewing the full text. After initial screening, references within the selected articles were reviewed to identify additional relevant articles. Duplicated articles with report on the same group of patients were considered once by including the most relevant or the latest article.

The two reviewers ZK and ZDY also independently extracted data using a prescribed form. The following data were extracted: first author's name, year of publication, study design, sample size, patients' clinical characteristics (age, sex, BMI), drug dose, treatment history and treatment duration, efficacy and safety outcomes. In case of discrepancies between the investigators during the process of article selection and data extraction, a third investigator would make the definitive decision.

Studies were through quality assessment by Jadad Scale [9]. The score contains 3 items: randomization, blinding, withdraws and dropouts. The lowest score is 0 and the highest score is 2 for the first 2 items and 1 for the third item. The total score of each study ranged from 0 to 5 .

\section{Statistical analysis}

As previous defined, SVR12 was used to estimate the efficacy. Safety outcomes included discontinuation of treatment related to therapy, AEs, serious adverse events (SAEs).

The main outcomes were expressed as dichotomous variables with $95 \%$ confidence interval $(\mathrm{CI})$. Heterogeneity among the studies was evaluated by Cochrane $\mathrm{Q}$ test with indices $I^{2}$ and $P$ value. The statistical significant level was set at 0.05 . If heterogeneity existed among the included studies, random effect model was used and Der Simonian and Laird method was used to calculate the pooled results [10]. Funnel plots were used to evaluate publication bias along with Egger's statistics [11]. Subgroup analyses and meta-regression analysis were also performed in this study. All the analyses were performed using Stata (version 13.0).

\section{Results}

\section{Characteristics of the included studies}

Seven studies met the inclusion and exclusion criteria were finally enrolled in this meta-analysis [12-18]. Figure 1 shows the flowchart illustrating the selection procedure. In total, 56 studies were identified through electronic database and manual searching. Five studies were duplicated and then removed. The titles and abstracts of the remaining articles were then screened. Among which 51 potentially eligible studies were selected for full-text and finally seven studies were included according to the predefined criteria.

Baseline characteristics of the participants were shown in (see Additional file 1: Table S1). A total of 1167 patients aged from 18 to 75 years co-infected with $\mathrm{HCV}$ and HIV were enrolled in our study, in which 948 patients were males and 147 patients have received prior treatment of $\mathrm{HCV}$. Overall 49 patients were infected with $\mathrm{HCV}$ genotype 1 and 318 patients were infected with $\mathrm{HCV}$ genotypes 2,3 and 4.

\section{Efficacy outcomes}

The pooled and subgroup analysis were shown in Fig. 2. Random-effect model was adopted as the $I^{2}$ was over $80 \%\left(\chi^{2}=53.73, P<0.01\right)$. The pooled SVR12 was $94.0 \%$ (95\%CI: 92.0\%-95.0\%). 


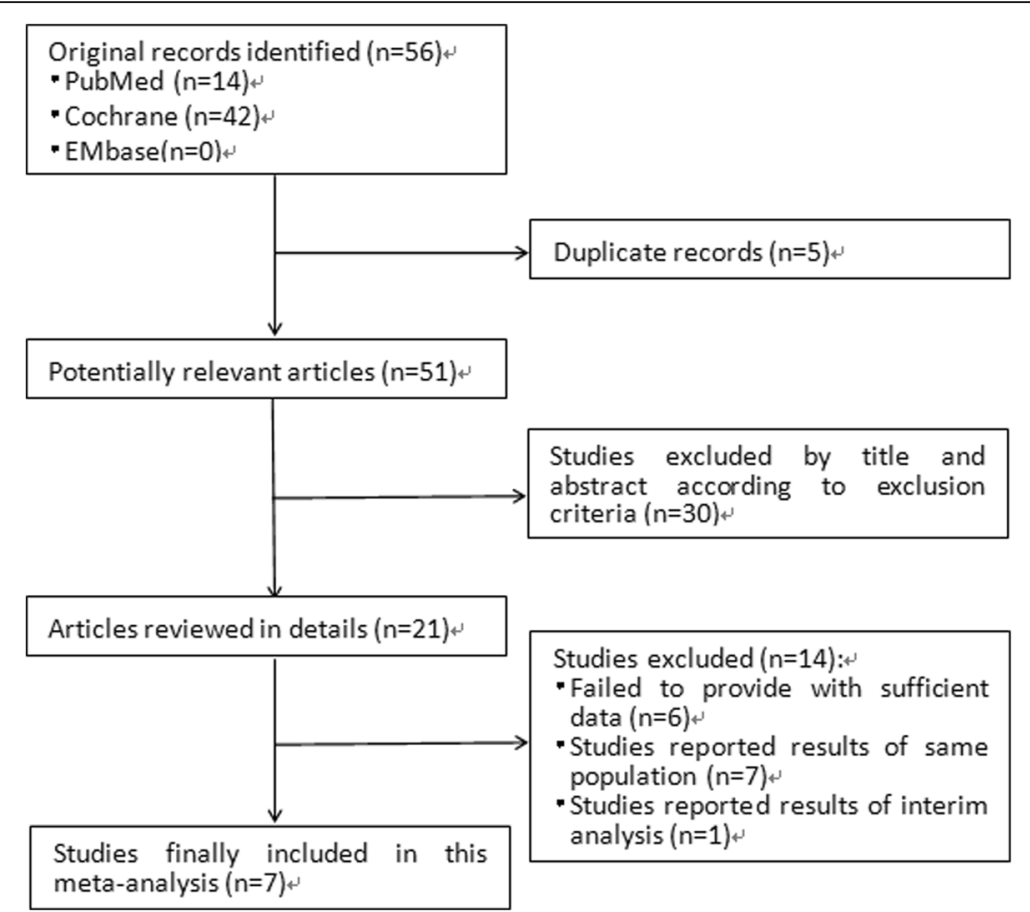

Fig. 1 Flow diagram of study selection

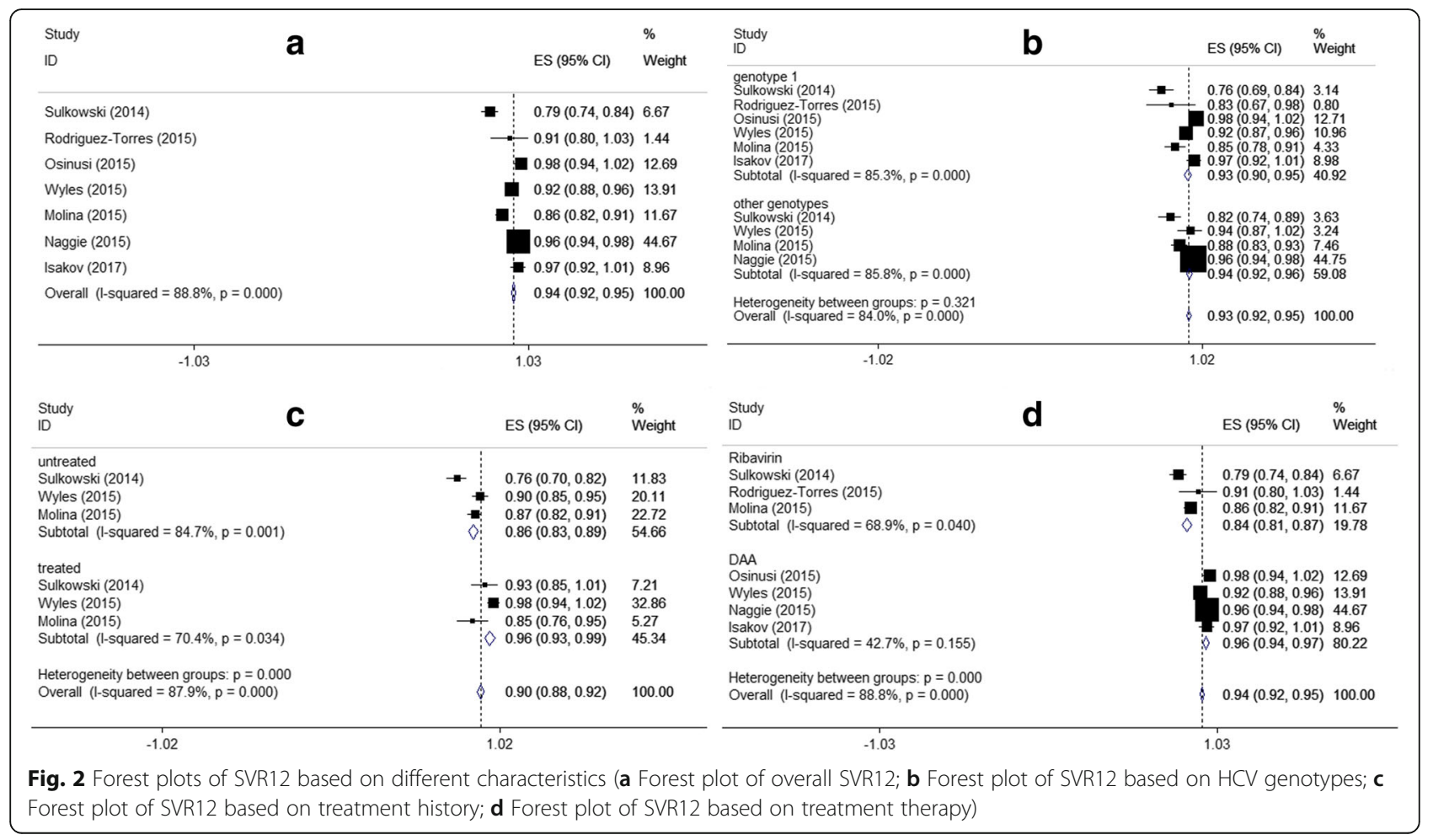


Based on treatment history, combined drugs, and $\mathrm{HCV}$ genotype, we subsequently performed subgroup analyses. The pooled SVR12 in patients infected with HCV genotype 1 and genotypes 2, 3 and 4 were $89.4 \%$ (95\%CI: $83.3 \%-95.6 \%)$ and $90.3 \%$ (83.6\%-96.9\%), respectively. The overall test of heterogeneity between patients infected with HCV genotype 1 and other types was not significant $(P=0.32)$. The results based on treatment history showed that the SVR12 in untreated patients was $86.0 \%$ (95\%CI: $83.0 \%-89.0 \%$ ), which was lower than that in treated patients with $96.0 \%$ (95\%CI: 93.0\%-99.0\%; $\left.X^{2}=21.39, P<0.01\right)$. Considering the combination therapy, the subgroup analysis showed that heterogeneity among patients using RBV and other DAAs both were not significant. Patients treated with SOF plus other DAAs had higher SVR12 than those treated with SOF and RBV.

\section{Safety outcomes}

By pooling the data from five of the seven studies, we observed that the heterogeneity of incidence of discontinuation and SAEs was not significant $\left(\chi^{2}=0.42, P=0.52\right.$; $\left.X^{2}=4.90, P=0.18\right)$. Pooled estimates of the rate of discontinuation and SAEs were $2.5 \%$ (95\%CI: $1.2 \%-3.9 \%)$ and 2.8\% (95\%CI: $1.4 \%-4.3 \%$ ), respectively (Fig. 3 ).

The most common AEs were fatigue, insomnia, asthenia, headache, diarrhea and nausea. Pooled results from six of the seven studies revealed high heterogeneity. The pooled incidence of any AEs was 67.8\% (95\%CI: 52.8\%-82.8\%).
Significantly higher occurrence rates of AEs might be found in the therapy with RBV $(89.0 \%, 95 \%$ CI: 86.0\%-93.0\%; $\left.\chi^{2}=55.17, P<0.01\right)$ (Fig. 3).

\section{Publication bias}

The funnel plot for SVR12 was shown in Fig. 4. Studies distributes closely within the $95 \%$ confidence interval axis, which indicated no obvious publication bias. In addition, the Egger's test for evaluating publication bias also showed no statistical significance $(t=-1.22$, $P=0.28)$.

\section{Meta-regression analysis}

The regression analysis was performed adjusting age, sex, BMI, HCV genotype and the combination drug. Finally we found the proportion of HCV genotype $1(t=$ 4.65, $P<0.01)$ and the combination drug $(t=3.91, P=$ 0.01 ) were with statistical significance, which could explain the heterogeneity. The Bubble plot was shown in Fig. 5.

\section{Discussion}

In this comprehensive meta-analysis of seven studies evaluating the efficacy and safety of SOF for treatment in $\mathrm{HCV} / \mathrm{HIV}$ co-infected patients, we found that the SVR12 was $94.0 \%$ (95\%CI: 92.0\%-95.0\%) and the rate of any AEs was 67.8\% (95\%CI: 52.8\%-82.8\%).

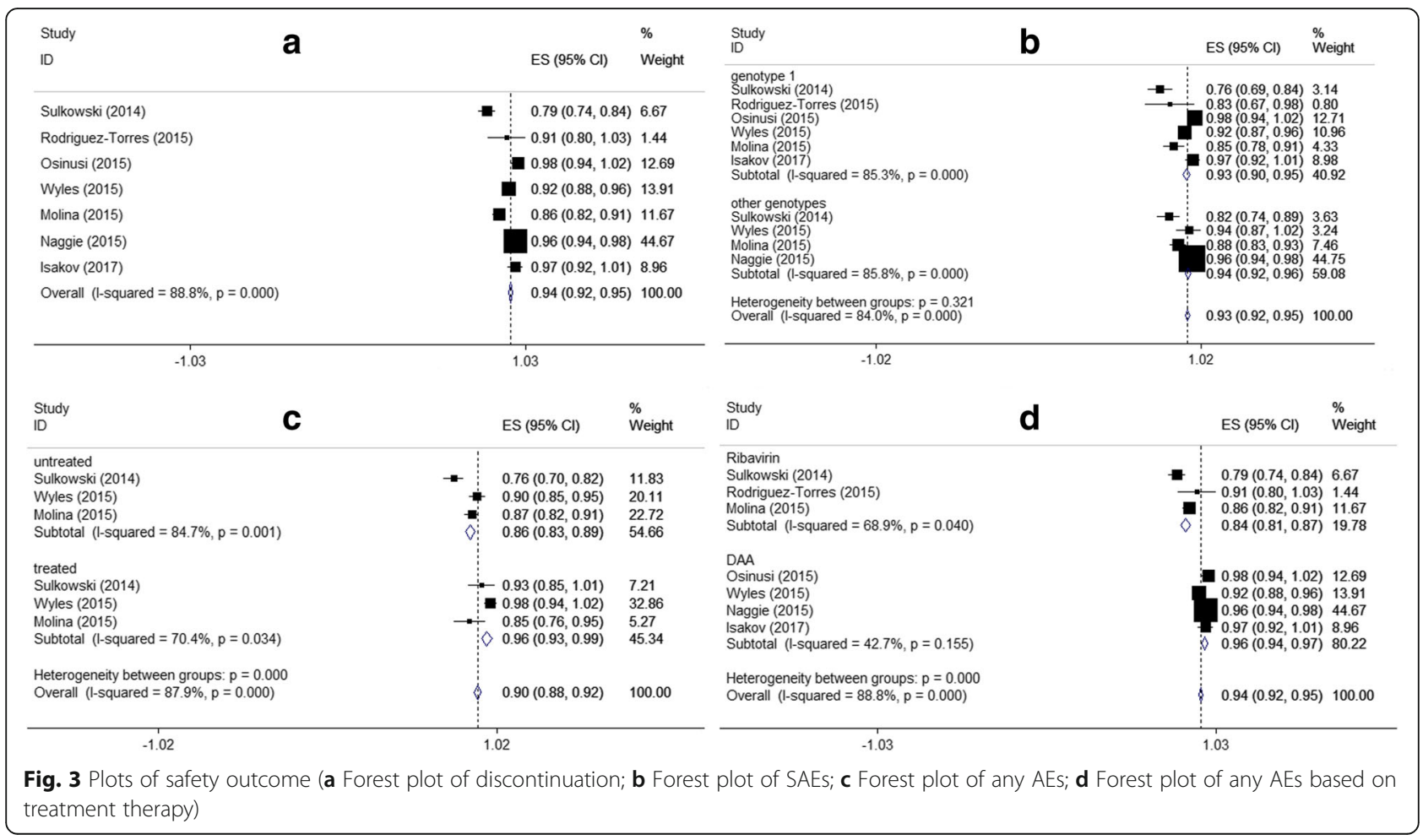




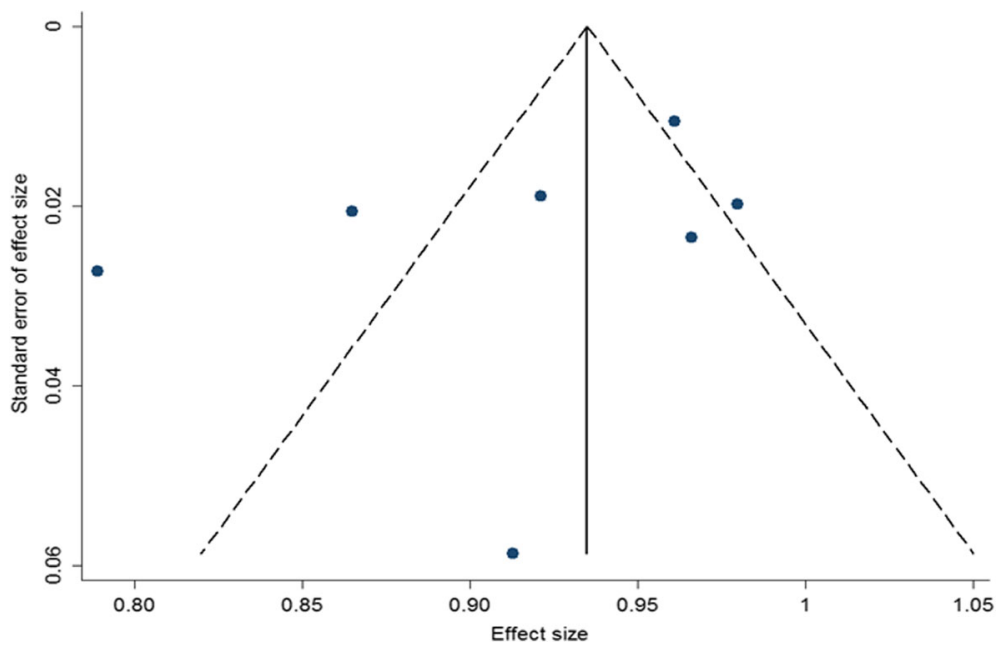

Fig. 4 Funnel plot of SVR12

Previous studies have summarized the drug-drug interaction between HCV and HIV treatments [19-21]. However, those reviews did not provide an overall estimation of the efficacy and safety of DAAs for treatment of HCV in $\mathrm{HCV} / \mathrm{HIV}$ co-infected patients. SOF with its all $\mathrm{HCV}$ genotype activity and high barrier of resistance [22] is becoming an attractive target for anti-HCV treatment, while the effect in $\mathrm{HCV} / \mathrm{HIV}$ co-infected patients was evaluated in some certain patients. This present study firstly attempt to review associated studies and provide a comprehensive analysis considering genotypes, treatment history, treatment regimen and other characteristics.

Although the heterogeneity was significant, it can be accounted for different combination therapies through subgroup analysis. The inconsistent conclusions in Sulkowski study [12] may be owing to its enrollment of few patients with very low CD4 cell count. In addition, in that study patients with cirrhosis (10\%) and women (17\%) were under presented. Subgroup analysis based on combination therapy found that patients with treatment of SOF and other DAAs showed higher SVR12 and lower occurrence of AEs than those treated with SOF and RBV. Meta-regression analysis also indicated the cause of heterogeneity.

Taking into consideration all the complex issues surrounding treatment in $\mathrm{HCV} / \mathrm{HIV}$ co-infected patients, it is important to ensure that all therapy are documented and scrutinized for potential drug-drug interactions and side effects. Also it is important that patients are managed in centers with experience in managing $\mathrm{HCV} / \mathrm{HIV}$ co-infected patients.

The strength of this meta-analysis lies in its exhaustive literature research, comprehensive statistical analysis and no significant evidence of publication bias. And the present study is the first research aiming at evaluating a drug in HCV/HIV co-infected patients.

This study has several limitations. First, most of the studies included in the analysis were uncontrolled trials due to the special management of HIV patients. Thus the single-arm trials limit the ability to derive definitive conclusions regarding the safety and efficacy of this regimen. Second, the original study designs slightly differing eligibility criteria of patients and treatment schedules may cofound the final results. All of these factors may lead to
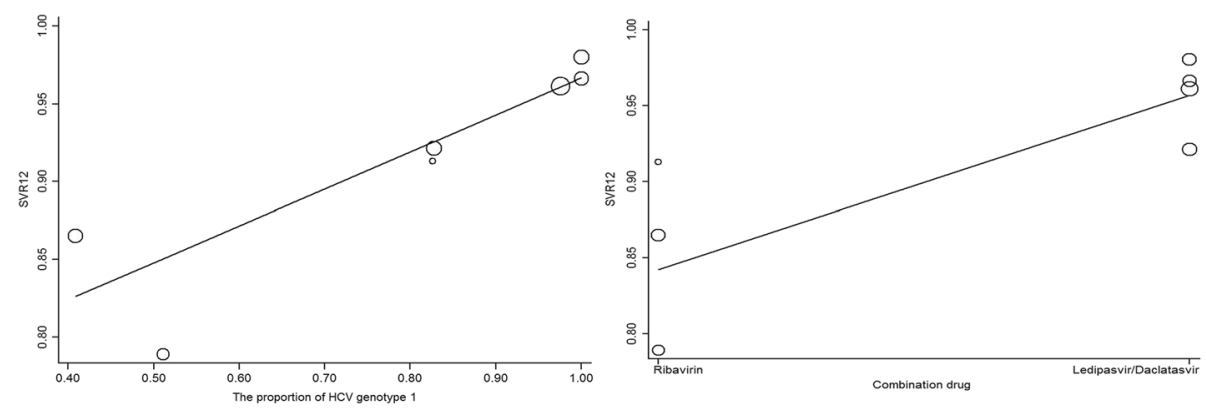

Fig. 5 Bubble plots of SVR12 
heterogeneity and therefore affect the estimates. Third, we did not conduct cost-effective analysis to assess the benefit and availability in clinical practice.

\section{Conclusion}

In summary, the present meta-analysis suggests that SOF containing regimen has shown positive effect for the treatment of $\mathrm{HCV} / \mathrm{HIV}$ co-infected patients, especially in those treatment-naive patients. Compared to RBV, patients treated with SOF combined with other DAAs had higher SVR12 and less AEs. Further large-scale, high quality and better designed clinical trials are needed to assess the combination of SOF and other DAAs based therapy.

\section{Additional file}

Additional file 1: Table S1. Baseline characteristics of participants. Table S1 shows the information of selected articles and the baseline characteristics of participants. (XLS $16 \mathrm{~kb}$ )

\section{Abbreviations}

AEs: Adverse events; DAAs: Direct acting antivirals; HCV: Hepatitis C virus; HIV: Human immunodeficiency virus; NS5B: Non-structural 5B; RBV: Ribavirin; SAEs: Serious adverse events; SOF: Sofosbuvir; SVR12: Sustained virological response

\section{Acknowledgements}

The authors are very thankful to Mr.Hu for helpful consultation and comments on this manuscript.

\section{Funding}

This study was supported by grants from the National Natural Science Foundation of China [grant number 81573213], and the Natural Science Foundation of Jiangsu Province, China [grant number BK20151089].

\section{Availability of data and materials}

The datasets supporting the conclusions of this article is included within the article and its additional files.

\section{Authors' contributions}

XZD and GTL designed the study and would take responsibility for all the entire process. KZ and DYZ performed the literature research and data collection; GQZ analyzed the data. GTL drafted the paper. All authors have read and approved the final paper

\section{Ethics approval and consent to participate}

Not applicable.

\section{Consent for publication}

Not applicable.

\section{Competing interests}

The authors declare that they have no competing interests.

\section{Publisher's Note}

Springer Nature remains neutral with regard to jurisdictional claims in published maps and institutional affiliations.

\section{Author details}

'Department of Infectious Diseases, LuoYang Central Hospital Affiliated to ZhengZhou University, Luoyang, Henan 471000, China. ${ }^{2}$ HuaDong Research Institute for Medicine and Biotecnics, No.293 Zhongshan East Road, Nanjing 210002, China.
Received: 9 August 2017 Accepted: 15 January 2018

Published online: 19 January 2018

\section{References}

1. Soriano V, Vispo E, Labarga P, Medrano J, Barreiro P. Viral hepatitis and HIV co-infection. Antivir Res. 2009;85(1):303-15.

2. Weber R, Ruppik M, Rickenbach M, Spoerri A, Furrer H, Battegay M, Cavassini M, Calmy A, Bernasconi E, Schmid P. Decreasing mortality and changing patterns of causes of death in the Swiss HIV cohort study. HIV Med. 2013; 14(4):195

3. Pascual Pareja JF, Montes ML, Gonzálezgarcía J, Arribas JR. Spontaneous viral clearance, viral load, and genotype distribution of hepatitis C virus (HCV) in European HIV-infected patients with anti-HCV antibodies. J Infect Dis. 2009; 199(10):1337-44.

4. Rockstroh JK, Mocroft A, Soriano V, Tural C, Losso MH, Horban A, Kirk O, Phillips A, Ledergerber B, Lundgren J. Influence of hepatitis C virus infection on HIV-1 disease progression and response to highly active antiretroviral therapy. J Infect Dis. 2005;192(6):992.

5. Berenguer J, Rodríguez E, Miralles P, Wichmann MAV, López-Aldeguer J, Mallolas J, Galindo MJ, Eynde EVD, Téllez MJ, Quereda C. Sustained Virological response to interferon plus Ribavirin reduces non-liver-related mortality in patients Coinfected with HIV and hepatitis C virus. Clin Infect Dis. 2012;55(5):728-36.

6. Rockstroh JK, Bhagani S. Managing HIV/hepatitis C co-infection in the era of direct acting antivirals. BMC Med. 2013;11(1):234.

7. Lam AM, Murakami E, Espiritu C, Steuer HMM, Niu C, Keilman M, Bao H, Zennou V, Bourne N, Julander JG. PSI-7851, a Pronucleotide of -D-2J-Deoxy2J-Fluoro-2J-C-Methyluridine Monophosphate, is a potent and pan-genotype inhibitor of hepatitis C virus Replication. Antimicrob Agents Chemother. 2010; 54(8):3187-96

8. International C. Recommendations for testing, managing, and treating hepatitis C. Recommendations for Testing Managing \& Treating Hepatitis C. 2014.

9. Jadad AR, Moore RA, Carroll D, Jenkinson C, Reynolds DJ, Gavaghan DJ, Mcquay HJ. Assessing the quality of reports of randomized clinical trials: is blinding necessary? Control Clin Trials. 1996;17(1):1-12.

10. Dersimonian R, Laird N. Meta-analysis in clinical trials. Control Clin Trials. 1986;7(3):177-88.

11. Stuck AE, Rubenstein LZ, Wieland D. Bias in meta-analysis detected by a simple, graphical test. Asymmetry detected in funnel plot was probably due to true heterogeneity. BMJ Clin Res. 1998;316(7129):469; author reply 470.

12. Sulkowski MS, Naggie S, Lalezari J, Fessel WJ, Mounzer K, Shuhart M, Luetkemeyer AF, Asmuth D, Gaggar A, Ni L, et al. Sofosbuvir and ribavirin for hepatitis C in patients with HIV coinfection. JAMA. 2014;312(4):353-61.

13. Rodriguez-Torres M, Gaggar A, Shen G, Kirby B, Svarovskaia E, Brainard D, Symonds WT, McHutchison JG, Gonzalez M, Rodriguez-Orengo J. Sofosbuvir for chronic hepatitis $C$ virus infection genotype 1-4 in patients coinfected with HIV. J Acquir Immune Defic Syndr. 2015;68(5):543-9.

14. Osinusi A, Townsend K, Kohli A, Nelson A, Seamon C, Meissner EG, Bon D, Silk R, Gross C, Price A, et al. Virologic response following combined ledipasvir and sofosbuvir administration in patients with HCV genotype 1 and HIV co-infection. JAMA. 2015;313(12):1232-9.

15. Wyles DL, Ruane PJ, Sulkowski MS, Dieterich D, Luetkemeyer A, Morgan TR, Sherman KE, Dretler R, Fishbein D, Gathe JC Jr, et al. Daclatasvir plus Sofosbuvir for HCV in patients Coinfected with HIV-1. N Engl J Med. 2015; 373(8):714-25.

16. Molina J-M, Orkin C, Iser D, Zamora F-X, Nelson M, Stephan C, Massetto B, Gaggar A, Ni L, Svarovskaia E, et al. Sofosbuvir plus ribavirin for treatment of hepatitis C virus in patients co-infected with HIV (PHOTON-2): a multicentre, open-label, non-randomised, phase 3 study. Lancet. 2015;385:1098-106.

17. Naggie S, Cooper C, Saag M, Workowski K, Ruane P, Towner WJ, Marks K, Luetkemeyer A, Baden RP, Sax PE, et al. Ledipasvir and Sofosbuvir for HCV in patients Coinfected with HIV-1. N Engl J Med. 2015:373(8):705-13.

18. Isakov V, Gankina N, Salupere R, Chulanov V, Kozhevnikova G, Zilmer K, Romanova S, Geyvandova N, Shimonova T, Gogova L, et al. Ledipasvir/ Sofosbuvir for 8 weeks results in high SVR rates in treatment-naive patients with chronic HCV infection and HIV/HCV co-infection. In: Hepatology conference: 67 th annual meeting of the american association for the study of liver diseases: the liver meeting 2016 United states conference start: 20161111 conference end: 20161115, vol. 63; 2017. p. 1010a. 
19. Mogalian E, Stamm L, Osinusi A, Shen G, Sajwani K, McNally J, Ling J, Mathias A. Drug interaction studies between sofosbuvir/velpatasvir and boosted HIV ARV regimens. In: Topics in antiviral medicine conference: $23 \mathrm{rd}$ conference on retroviruses and opportunistic infections, CROI 2016 United states conference start: 20160222 conference end: 20160225, vol. 24; 2017. p. 40.

20. Karageorgopoulos DE, El-Sherif O, Bhagani S, Khoo SH. Drug interactions between antiretrovirals and new or emerging direct-acting antivirals in HIV/ hepatitis C virus coinfection. Curr Opin Infect Dis. 2014;27(1):36.

21. Mogalian E, Stamm L, Osinusi A, Shen G, Sajwani K, McNally J, Mathias A. Drug-drug interaction studies between HCV antivirals sofosbuvir and GS-5816 and HIV Antiretrovirals. In: Hepatology (varpagings), vol. 62; 2015. p. 1310a.

22. Sofia MJ, Bao D, Chang W, Du J, Nagarathnam D, Rachakonda S, Reddy PG, Ross BS, Wang $P$, Zhang HR. Discovery of a $\beta-d-2^{\prime}$-deoxy-2'-a-fluoro-2'- $\beta-C$ methyluridine nucleotide prodrug (PSI-7977) for the treatment of hepatitis C virus. J Med Chem. 2010:53(19):7202-18.

Submit your next manuscript to BioMed Central and we will help you at every step:

- We accept pre-submission inquiries

- Our selector tool helps you to find the most relevant journal

- We provide round the clock customer support

- Convenient online submission

- Thorough peer review

- Inclusion in PubMed and all major indexing services

- Maximum visibility for your research

Submit your manuscript at www.biomedcentral.com/submit 